Check for updates

Cite this: Chem. Sci., 2019, 10, 6322

๑ All publication charges for this article have been paid for by the Royal Society of Chemistry

Received 19th March 2019

Accepted 10th May 2019

DOI: $10.1039 / c 9 s c 01345 h$

rsc.li/chemical-science

\section{Vinylphosphonites for Staudinger-induced chemoselective peptide cyclization and functionalization $\uparrow$ t:}

\author{
Marc-André Kasper, $\S^{\mathrm{ab}}$ Maria Glanz, $\S^{\mathrm{ab}}$ Andreas Oder, ${ }^{a}$ Peter Schmieder, ${ }^{a}$ Jens P. von \\ Kries $^{a}$ and Christian P. R. Hackenberger (D) *ab
}

\begin{abstract}
In this paper, we introduce vinylphosphonites for chemoselective Staudinger-phosphonite reactions (SPhR) with azides to form vinylphosphonamidates for the subsequent modification of cysteine residues in peptides and proteins. An electron-rich alkene is turned into an electron-deficient vinylphosphonamidate, thereby inducing electrophilic reactivity for a following thiol addition. We show that by varying the phosphonamidate ester substituent we can fine-tune the reactivity of the thiol addition and even control the functional properties of the final conjugate. Furthermore, we observed a drastic increase in thiol addition efficiency when the SPhR is carried out in the presence of a thiol substrate in a one-pot reaction. Hence, we utilize vinylphosphonites for the chemoselective intramolecular cyclization of peptides carrying an azide-containing amino acid and a cysteine in high yields. Our concept was demonstrated for the stapling of a cell-permeable peptidic inhibitor for proteinprotein interaction (PPI) between BCL9 and beta-catenin, which is known to create a transcription factor complex playing a role in embryonic development and cancer origin, and for macrocyclization of cellpenetrating peptides (CPPs) to enhance the cellular uptake of proteins.
\end{abstract}

\section{Introduction}

Selective chemical modification of the sulfhydryl group of cysteine is an important tool in the life sciences. ${ }^{1,2}$ Cysteineselective reactions have been employed to covalently modify proteins in a residue specific manner for various applications including drug delivery, ${ }^{3}$ incorporation of post-translational modifications ${ }^{4}$ and activity based protein profiling. ${ }^{5}$

A plethora of different techniques have been developed in the past few decades that allow for selective modification of cysteine residues on peptides and proteins based on various reaction mechanisms. Michael additions to $\alpha, \beta$-unsaturated carbonyl compounds in maleimides, ${ }^{6}$ sulfone reagents ${ }^{7}$ and recently developed carbonylacrylic reagents ${ }^{8}$ are the most prominent examples. Other methods rely on $\mathrm{SN}_{2}$-reactions of haloalkyl bonds such as iodoacetamides, ${ }^{9}$ on alkynylation ${ }^{10}$ and fluoroalkylation $^{11}$ with hypervalent iodine reagents, on

${ }^{a}$ Leibniz-Forschungsinstitut für Molekulare Pharmakologie (FMP), Chemical Biology Department, Robert-Rössle-Strasse 10, 13125 Berlin, Germany. E-mail: hackenbe@ fmp-berlin.de

${ }^{b}$ Humboldt Universität zu Berlin, Department of Chemistry, Brook-Taylor-Str. 2, 12489 Berlin, Germany

$\dagger$ This work is dedicated to Prof. Ron Raines on the occasion of his $60^{\text {th }}$ birthday. \$ Electronic supplementary information (ESI) available. See DOI: 10.1039/c9sc01345h

$\S$ These authors contributed equally. nucleophilic aromatic substitution of perfluoroaromatic compounds ${ }^{12}$ or on Pd-mediated cross-coupling. ${ }^{13}$ The level of reactivity for cysteine in these methods is predetermined by the chemical nature of the reactive group itself. Alteration of this reactivity is often difficult and requires tedious chemical manipulations. However, certain applications such as activity based protein profiling ${ }^{14}$ or covalent enzyme inhibition ${ }^{15}$ often rely on reduced reactivity that allows for modification of the active site cysteine while sparing all other free nucleophiles in the proteome. Such decreased reactivity has for instance been achieved by substituting the highly reactive iodide in $\alpha$-haloacetamides with a less reactive chloride ${ }^{16}$ Therefore, a cysteine selective modification method that utilizes easily tunable electrophilic warheads would be highly attractive for addressing challenges in peptide and protein chemistry.

Aside from protein modification, cysteine selective reactions have also been applied to peptide synthesis. One or two covalent linkages between cysteine residues in peptides enable the construction of macro or bicyclized peptides with enhanced biophysical and pharmacological properties. ${ }^{17}$ Upon macrocyclization, peptidic structures can become more stable against proteases ${ }^{18}$ they can be locked in their structural conformation by covalently linking side chains and therefore restricting their conformational flexibility ${ }^{19,20}$ or increasing their function by rigid constraint as shown for various cyclic bioactive ${ }^{21}$ or cell penetrating peptides (cCPPs). ${ }^{22}$ The groups of Pentelute and Derda introduced perfluoroaromatic linkages 
for the cyclization of peptides containing two cysteines. ${ }^{23,24}$ Tricyclic peptides have also been synthesized by alkylation with tris-(bromomethyl)benzene ${ }^{25}$ and very recently by the incorporation of two chemical bridges with a set of various dibromoalkyl reagents. ${ }^{26}$ Such chemoselective modification strategies are advantageous over common cyclization techniques $^{27}$ such as lactamization, ${ }^{28}$ since they can be applied after cleavage from the resin and preclude therefore tedious orthogonal protecting group strategies during SPPS. In addition, even cysteine containing proteins can be cyclized by applying chemoselective techniques, as recently demonstrated with the aid of a trimeric chloroacetamide. ${ }^{29}$

Based on the Staudinger-phosphonite reaction (SPhR), developed in our laboratory, ${ }^{30}$ we recently introduced ethynylphosphonamidates as cysteine-selective electrophiles that can be chemoselectively incorporated starting from ethynylphosphonites. ${ }^{31}$ In the current paper, we report vinylphosphonites as a new compound class for the transformation of azides into electron deficient alkenes that selectively modify cysteine residues in peptides and proteins. We observed that the SPhR with vinylphosphonites facilitates straightforward access to vinylphosphonamidates carrying different $O$-substituents. We discovered that the rate of the subsequent thiol addition can be drastically influenced by the electronic properties of these phosphonamidate ester substituents. In addition, we engineered an intramolecular variant of the chemically induced thiol addition for a chemoselective cyclization of peptides. We were able to demonstrate that azido modified cysteine peptides can be cyclized upon SPhR with vinylphosphonites in high yields (Scheme 1). In contrast to other chemoselective peptide cyclizations, which have been mainly used for head-to-tail cyclizations, including traceless Staudinger ligation, ${ }^{32} \alpha$-ketoacid-hydroxylamine amide-ligation ${ }^{33}$ or serine ligation, ${ }^{34}$ the method presented herein can be straightforwardly applied to side-chain ring closure.

\section{Results and discussion}

Previously, Gao et al. have observed that methyl-substituted vinylphosphonamidates are unreactive in base mediated thiol additions, ${ }^{35}$ probably due to decreased electrophilicity, compared to their ethynyl substituted counterparts. ${ }^{31} \mathrm{We}$ anticipated that the electrophilic reactivity of the double bond towards thiol-addition can be increased by attaching different electron-withdrawing substituents to the phosphonamidate ester moiety. Since this residue is preserved during SPhR with azides, we started our investigations by synthesizing different substituted vinylphosphonites. Here, we followed previously published procedures starting from either phosphorus trichloride (route I) or bis(diisopropylamino)-chlorophosphine (route II). ${ }^{36}$ Several attempts to isolate unprotected vinylphosphonites failed due to product decomposition, hence we decided to isolate the phosphonites in their borane-protected form and use them directly as starting materials for the subsequent SPhR. Vinylphosphonite borane adducts proved to be stable during column chromatography and we were able to obtain four vinylphosphonites with ethyl, 1a, phenyl, $\mathbf{1 b}$ and electron withdrawing 2-nitrobenzyl, 1c, and trifluoroethyl, 1d, substituents (Fig. 1a).

With vinylphosphonites 1a-d in hand, we continued our studies to perform the SPhR with two different model azides 2 and 3 to synthesize both $N$-phenyl $\mathbf{4 a - d}$ and $N$-alkyl substituted vinylphosphonamidates $\mathbf{5 a - d}$. Here, we applied previously published procedures, either diethyl chlorophosphite alkylation with vinylmagnesium bromide ${ }^{31}$ (route III) or borane deprotection with DABCO in toluene ${ }^{37}$ (route IV), directly followed by azide addition and hydrolysis without isolation of the phosphonite intermediate. The SPhR was always carried out in dry solvents to prevent hydrolysis of the phosphonites. All of the tested phosphonites reacted smoothly under the tested conditions and the desired vinylphosphonamidates could be isolated by column chromatography. Electron withdrawing substituents were well tolerated in the reaction with the aryl azide 2 and with the alkyl azide 3 (Fig. 1 b).

Next, we probed the reactivity of the synthesized vinylphosphonamidates towards thiol addition in aqueous solution under slightly basic conditions as previously performed for ethynylphosphonamidates ${ }^{31}$ (Fig. 2a). For this, a time course for the addition of glutathione (GSH) to vinylphosphonamidates 4a-d and 5a-d in ammonium bicarbonate buffer was recorded.

Under the tested conditions $(10 \mathrm{mM}$ phosphonamidate and two equivalents of GSH) the $N$-phenyl vinylphosphonamidate 4a showed a good conversion of $80 \%$ to the desired thiol adduct after $24 \mathrm{~h}$. In contrast to $\mathbf{4 a}$, the comparable $N$-alkyl derivative $\mathbf{5 a}$ was only converted $20 \%$ under the same conditions (Fig. 2b), which is in accordance with the observations of Gao et al. on the unreactivity of $\mathrm{N}$-alkyl-O-methyl-vinylphosphonamidate towards thiols; however, the previously mentioned dealkylation of the phosphonamidate ester was not observed under conditions applied here. Since we assumed that such slow kinetics would limit the applicability to protein modification, for example by undesired disulfide formation of the peptide or protein substrate, we also tested compounds with electron-
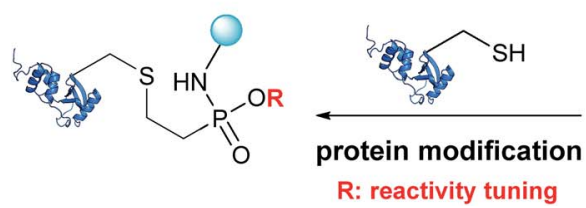
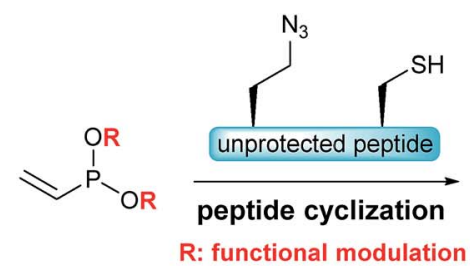

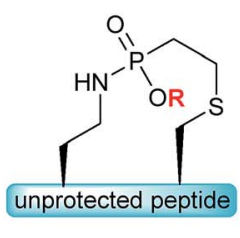

Scheme 1 Vinylphosphonites for intramolecular peptide cyclization and cysteine selective protein modification. 
a)

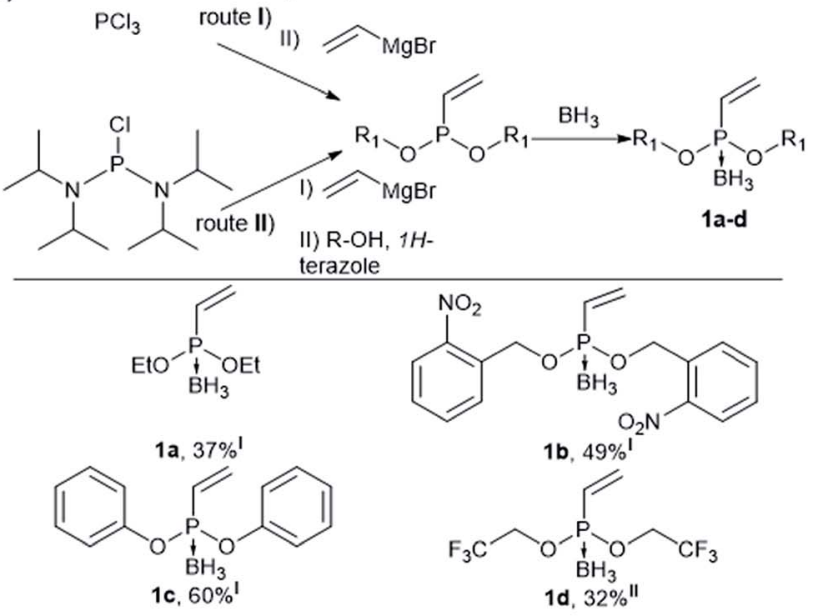

b)
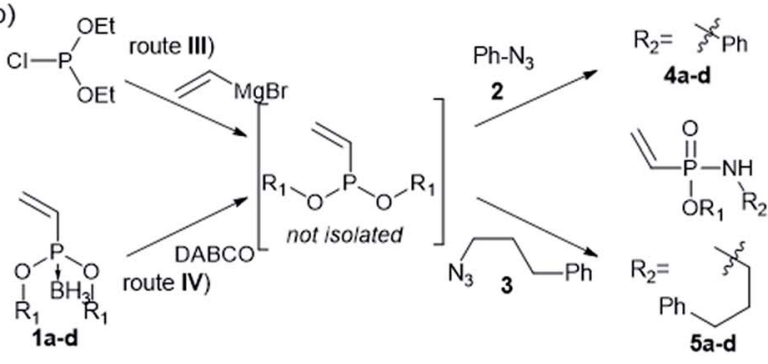

Fig. 1 (a) Synthesis of substituted vinylphosphonites from phosphorus trichloride (route I) or bis(diisopropylamino)chlorophosphine (route II). Stated are isolated yields. (b) One-pot SPhR with vinylphosphonites and azides. Synthesis from borane protected vinylphosphonites (route III) or diethyl chlorophosphite (route IV). Stated are isolated yields.

deficient phosphonamidate ester substituents in the addition of glutathione. A conversion of $50 \%$ is reached after two and a half hours for 2-nitrobenzyl compound $\mathbf{4 b}$ and after only 30 minutes for trifluoroethyl substituted $\mathbf{4 d}$. The $O$-phenyl compound $\mathbf{4 c}$ shows an accelerating effect over alkoxy-substituents as well, reaching $50 \%$ conversion in only one hour (Fig. 2c). These

a)

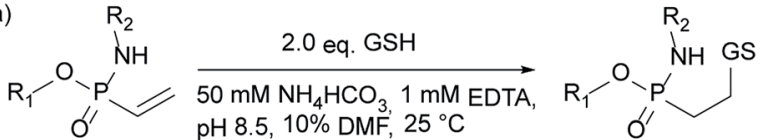

$\mathrm{R}_{1}=$ ethyl, 2-nitrobenzyl, phenyl, trifluoroethyl

$\mathrm{R}_{2}=$ phenyl, 3-phenylpropyl

b)
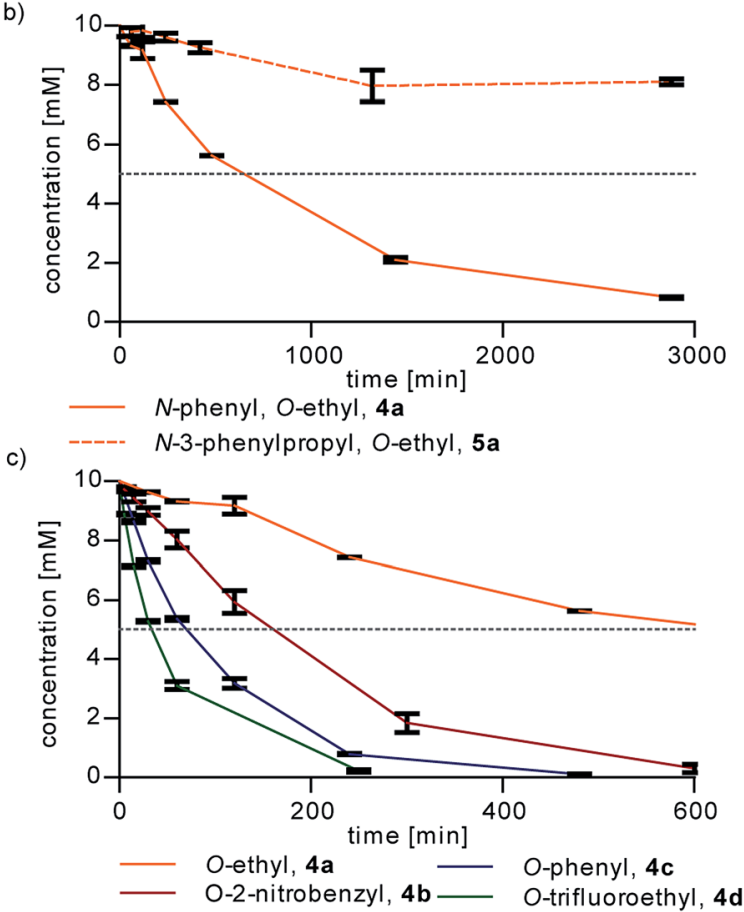

Fig. 2 (a) Reaction of glutathione (GSH) with differently substituted vinylphosphonamidates. $10 \mathrm{mM}$ vinylphosphonamidates were reacted with $20 \mathrm{mM} \mathrm{GSH}$ at $\mathrm{pH} 8.5\left(\mathrm{NH}_{4} \mathrm{HCO}_{3}\right.$ buffer). Concentration of the starting materials over time, mean and error from three independent measurements, monitored by UPLC-UV. (b) N-Phenyl vs. $\mathrm{N}$-alkyl comparison. (c) Comparison of different $\mathrm{O}$-substituents. Dotted line depicts $50 \%$ conversion.

results clearly show that the reactivity of vinylphosphonamidates can be tuned by varying the phosphonamidate ester residue. The corresponding $N$-alkyl substituted compounds 5a-d also undergo rate enhancement with more electron withdrawing $O$-substituents, with a slower reaction compared to the $N$-phenyl substituted compounds $4 a-d$ in general (ESI Fig. S1 $\ddagger$ ). Notably, the glutathione adduct was the only detectable product by UPLC/MS and no other side product was observed for any of the tested phosphonamidates (ESI Fig. S2 ). The identity of the thiol-glutathione adducts of $4 a$ (ESI Fig. S3 ) and 4d was confirmed by NMR characterization after isolation by preparative HPLC in $60 \%$ and $89 \%$ yield, respectively.

Further kinetic investigations of the thiol addition to $4 \mathbf{d}$, being the fastest compound tested in this study (ESI Fig. S1 $\$$ ), revealed a second-order rate constant of $0.021 \mathrm{M}^{-1} \mathrm{~s}^{-1}$ (ESI Fig. S4). Even though this is considerably slower than that of maleimides $\left(734 \mathrm{M}^{-1} \mathrm{~S}^{-1}\right){ }^{38}$ ethynylphosphonamidates (0.62 $\left.\mathrm{M}^{-1} \mathrm{~s}^{-1}\right)^{31}$ and recently introduced dinitroimidazoles, ${ }^{39}$ the rate is still higher or in the range of other protein modification 
reactions such as oxime, Staudinger or Pictet-Spengler ligations. ${ }^{38}$ Next to reaction rates, an important aspect for many applications of cysteine selective protein modification reagents is susceptibility of the conjugate towards thiol exchange. ${ }^{1}$ Maleimides are in particular known to undergo retro Michael addition, followed by transfer of the modification to other cysteine containing molecules in solution. ${ }^{40}$ To prove the stability of the vinylphosphonamidate adducts in the presence of other thiols, we incubated the glutathione adducts of $\mathbf{4 a}$ and 4d with an excess of 100 equivalents of 2-mercaptoethanesulfonate. Even after one week of incubation, no thiol exchange could be observed for both of the constructs by UPLC/MS (ESI Fig. S5:).

Next, we wanted to probe the reactivity of electron-poor vinylphosphonamidates towards cysteine modification on antibodies. For this, we synthesized a fluorescent EDANSmodified vinylphosphonamidate $\mathbf{6}$, analogously to compound 4d, with the $N$-phenyl-O-trifluoroethyl substitution pattern that has been identified to be superior in thiol addition kinetics. Based on a previously established protocol ${ }^{31}$ we reduced the four interchain disulfide bonds of cetuximab to generate 8 free cysteine residues and probed the reactivity towards 6 (Fig. 3a). Successful labelling after reduction and incubation with 20 equivalents of reagent per antibody was confirmed by in-gel fluorescence (Fig. 3b). Intact protein MS revealed a decent

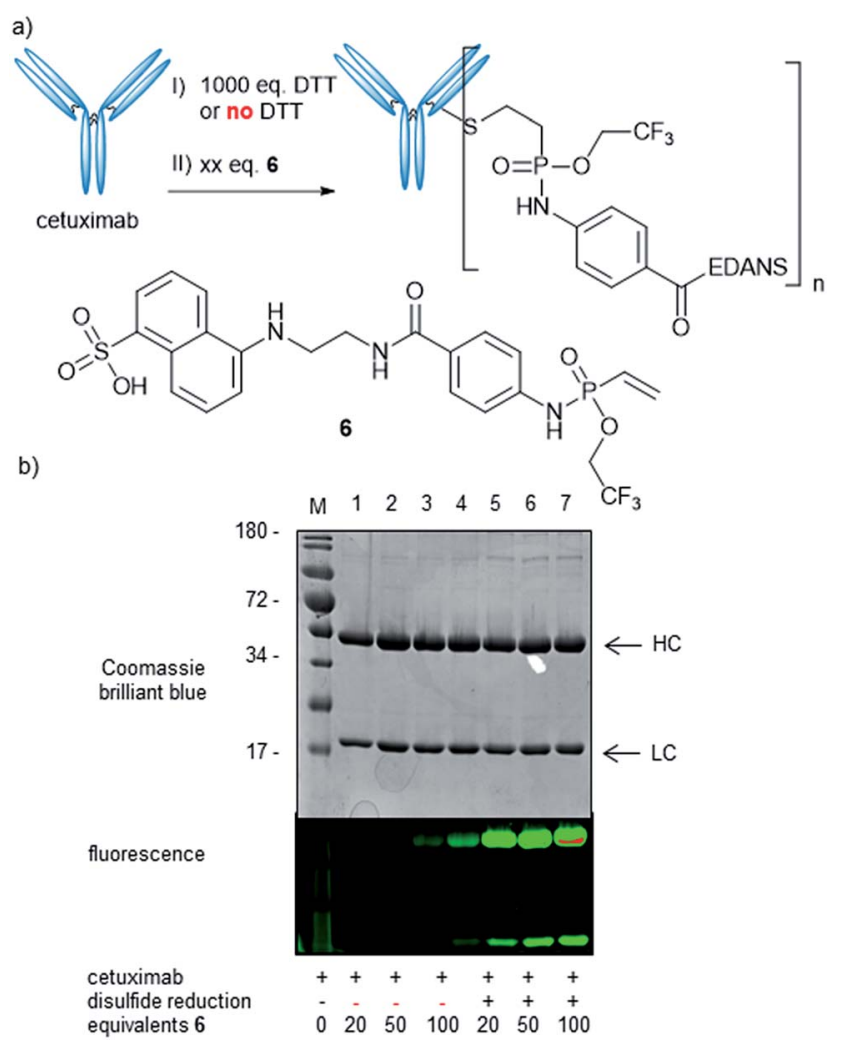

Fig. 3 Cetuximab modification with 6 . (a) Reduction and alkylation principles and structure of 6 . (b) Gel analysis: reactions were carried out with varying equivalents of 6: Lane 1: untreated antibody. Lane 24: control reactions without prior DTT treatment. Lane 5-7: prior DTT treatment. HC: antibodies' heavy chain, LC: light chain. degree of modification of 3.8 fluorophore molecules per antibody (ESI Fig. S6 ). To probe the cysteine selectivity, a control experiment without prior antibody reduction was performed and no modification could be detected after incubation with 20 equivalents of 6 by in-gel fluorescence and MS. Only upon exposure of the unreduced antibody to high reagent excesses of more than 50 equivalents per antibody, low degrees of modification were observed (Fig. 3b). The observation of unspecific labelling at higher reagent concentrations was further investigated by mass spectrometry (LC-MS/MS) after trypsin digestion of the reduced antibody modified with 100 eq. of 4d (ESI Fig. S7). In addition to the inter-chain disulfide forming cysteines, a few lysine residues were also found to be modified. This cross-reactivity with lysine is also known for other cysteine labelling reagents such as iodoacetamides ${ }^{41}$ and maleimides. ${ }^{42}$ The findings are nevertheless contrary to our previously reported ethynylphosphonamidate reagents that exhibit excellent cysteine selectivity even with large reagent excesses. ${ }^{31}$ To further broaden the scope of our method, we also synthesized a biotinmodified 2-nitrobenzyl-phosphonamidate with a structure that has the same substitution pattern as compound $\mathbf{4 b}$. Successful antibody labelling was confirmed by anti-biotin Western blot analysis. Analogously to the trifluoroethyl compound, only prolonged reaction times lead to minor labelling degrees without prior reduction of the antibody disulfide bonds (ESI Fig. S8:). Taken together, we were able to show that vinylphosphonamidates can lead to efficient and selective modification of cysteine residues on proteins using only slight reagent excesses.

We further anticipated that the SPhR with subsequent thiol addition could also be applied in an intramolecular reaction. Therefore we synthesized an azidohomoalanine- and cysteinecontaining peptide, aiming to initiate a covalent cyclization. As the first proof of principle study, we chose to macrocyclize the BCL9 derived peptide 8a, which has been reported to act as a potent PPI-inhibitor when stapled by ring closing metathesis (RCM) ${ }^{43}$ We decided to replace the two olefinic amino acids in the $i, i+4$ position with azidohomoalanine and cysteine in order to keep the linker length (10 atoms) similar to that of the literature-known peptide 7 (Scheme 2). In our initial experiments we aimed to keep a double bond in the linker structure for more rigidity and hence used an ethyl-substituted ethynylphosphonite in the reaction with peptide 8a in dry DMSO based on our previously published conditions. ${ }^{31}$ However, we found that in order to promote the initial SPhR with the alkylazide of azidohomoalanine the reaction temperature had to be raised to $50{ }^{\circ} \mathrm{C}$, upon which the macrocyclized product formed only in low amounts. A major side product observed by UPLC-UV/MS was a peptide with an additional $28 \mathrm{Da}$, which we attribute to the alkylation of the nitrogen of the phosphonamidate. Similar $N$-alkylated phosphoramidates were reported previously by us after the rearrangement of phosphorimidates under elevated temperatures. ${ }^{44}$ We hypothesized that raising the reactivity of the phosphonite by using a more nucleophilic vinyl- as opposed to the ethynyl-phosphonite in the SPhR enables the use of lower reaction temperature and would thereby prevent the rearrangement to the alkylated byproduct. Therefore purified 


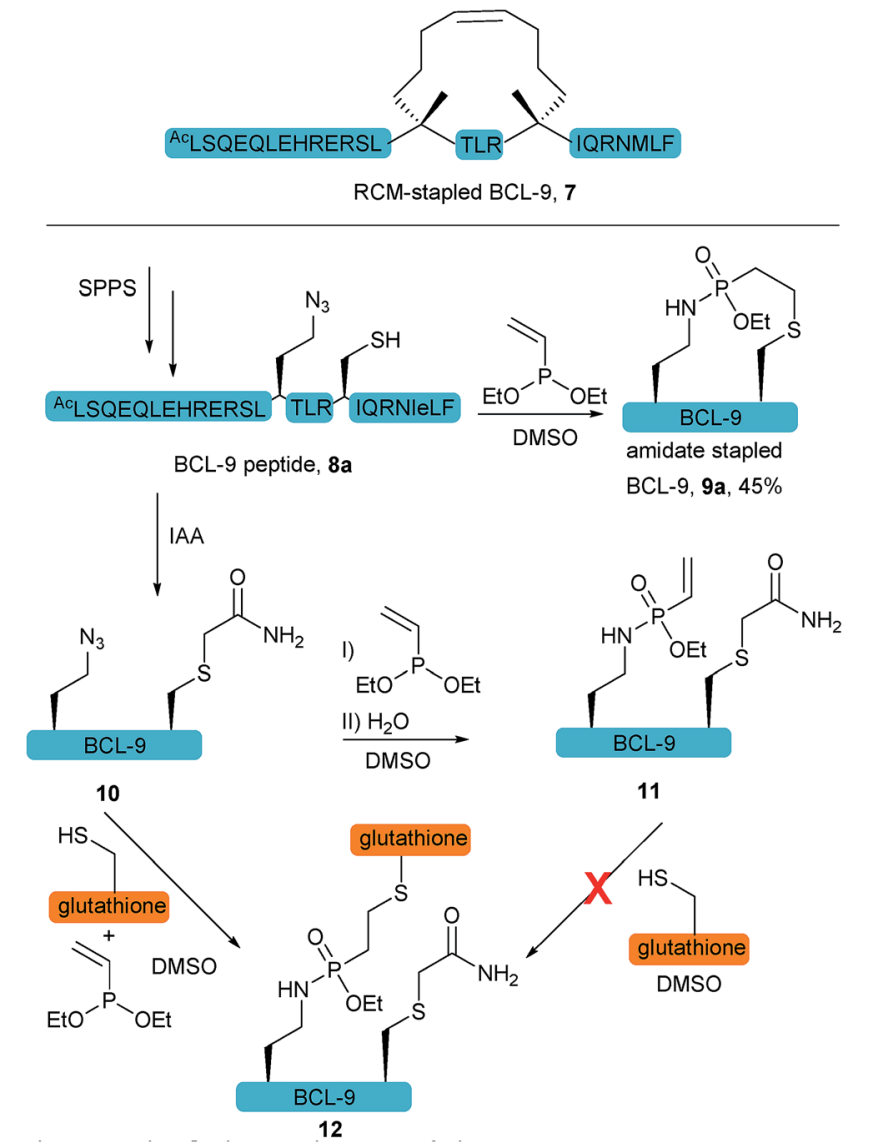

Scheme 2 Principle of the intramolecular peptide cyclization of an azido containing cysteine-BCL-9 derived peptide. Investigations with a capped cysteine peptide (9) revealed an accelerated thiol addition when SPhR is performed in the presence of thiols. SPhR on peptides is always carried out with the crude diethyl phosphonite, synthesized from diethyl chlorophosphite (route III).

peptide 8a was reacted with crude diethyl vinylphosphonite (route III) after SPPS in an over-night reaction in DMSO. To our delight, we isolated the desired cyclized peptide 9a after preparative HPLC as the main product in a very good yield of $45 \%$. This result is particularly remarkable in light of the slow intermolecular thiol addition to $O$-ethyl- $N$-alkylvinylphosphonamidates such as $\mathbf{5 a}$ as shown before (Fig. 2 b). UPLC/MS showed a clean conversion towards the desired product without significant amounts of the side product (ESI Fig. S9:).

To gain more insight into the in situ intramolecular cyclization, we capped the cysteine of peptide 8a with iodoacetamide to form peptide 10. This peptide was reacted with glutathione under two different reaction conditions (Scheme 2). First, glutathione was added in a one-pot reaction with peptide $\mathbf{1 0}$ $(1.65 \mathrm{mM})$ and 5 eq. diethyl vinylphosphonite. Here, the thiol addition product $\mathbf{1 2}$ could be observed by LC-MS as the major product (ESI Fig. S10 ). In the second experiment, we reacted peptide 10 with 5 eq. vinyl phosphonite to form the phosphonamidate $\mathbf{1 1}$ after hydrolysis, as observed by LC-MS. After lyophilization peptide $\mathbf{1 1}$ was incubated with 1 eq. glutathione
(1.65 mM) in DMSO for 24 hours. Under these conditions, no product formation could be observed by MS analysis (ESI Fig. S10 ). This experiment again points towards the decreased reactivity of ethyl-substituted vinylphosphonamidates in intermolecular reactions with thiol substrates (see Fig. 2a and ref. 35), and furthermore implies that the in situ process benefits from the reaction of thiols with a more reactive $\mathrm{SPhR}$ intermediate.

With the phosphonamidate-cyclized BCL9 peptide 9a in hand we set out to investigate its stability and helicity. At first, 9a was incubated at different $\mathrm{pH}$ values over several days and the stability monitored by UPLC. Under acidic conditions below $\mathrm{pH} 2$ the $\mathrm{P}-\mathrm{N}$-bond gets cleaved within 24 hours, which is in accordance with our previous observations. ${ }^{31}$ At neutral and basic $\mathrm{pH}$, the phosphonamidate moiety proved to be stable over several days without any observable decomposition (Fig. 4a and b). Next, we evaluated the peptides' helical propensities by CD spectroscopy and could show a drastic increase in helicity upon cyclization from $20 \%$ to around $50 \%$ (Fig. 4d). To verify the ability to disrupt the PPI between purified BCL9 and $\beta$-catenin we used a homogeneous assay with time resolved fluorescence (HTRF), in which both interacting proteins were recognized by distinct antibodies via orthogonal tags and conjugated fluorophores suitable for energy transfer. ${ }^{45}$ Disturbance of the protein-protein interaction causes a decreased FRET signal (ESI Fig. S11). Using this assay, we could show that the phosphonamidate cyclized peptides can disrupt the PPI at concentrations in the nM range (Fig. 4e). The $\mathrm{IC}_{50}$ values are almost identical to those reported for the RCM stapled BCL9 peptide. ${ }^{43}$ Furthermore, we observed that the stereochemistry at cysteine seems to have an influence on the disruption behavior, with a superior inhibition constant observed for the D-enantiomer $\mathbf{9 b}$ compared to that of the L-enantiomer 9a.

Since peptide stapling is known to improve cellular uptake through endocytic vesicle trafficking ${ }^{46}$ and the BCL-9 and $\beta$ catenin interaction takes place intracellularly in the cytoplasm and the nucleus, ${ }^{45}$ we also tested the peptides' ability to penetrate cells. By treating HeLa cells with $10 \mu \mathrm{M}$ mixture of $10 \%$ fluorescein labelled and $90 \%$ non-labelled peptides for three hours, we observed that peptide $\mathbf{9 b}$ shows almost no uptake, whereas the RCM stapled peptide 7 is taken up readily. We attributed this to a recent observation from the Futaki lab, in which they show the importance of net hydrophobicity for the cellular uptake of stapled peptides. ${ }^{47}$ In order to increase the hydrophobicity of our linkage, we synthesized peptide $\mathbf{1 3}$ with a more lipophilic $O$-benzyl-substituent at the phosphonamidate. For this, the SPhR of the azido peptide $\mathbf{8 b}$ with a benzyl phosphonite was carried out at slightly elevated temperatures to ensure high conversions. The peptide was only synthesized in the D-cysteine variant due to the aforementioned improved disruption behavior towards the BCL-9 and $\beta$-catenin interaction. Isolated 13 showed comparable helicity to the ethyl substituted 9b (Fig. 4d) and the benzylsubstituent did not hamper the peptides' ability to disrupt the PPI (Fig. 4e). In addition, we observed increased cellular uptake of benzyl substituted $\mathbf{1 3}$, compared to $\mathbf{9 b}$, which nicely 
a)

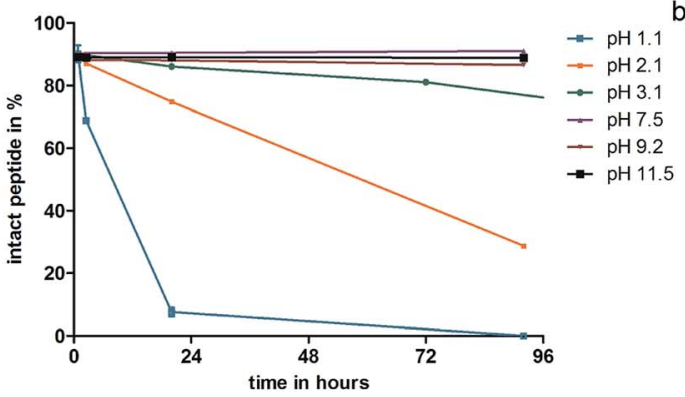

c)

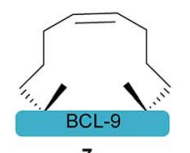

d)

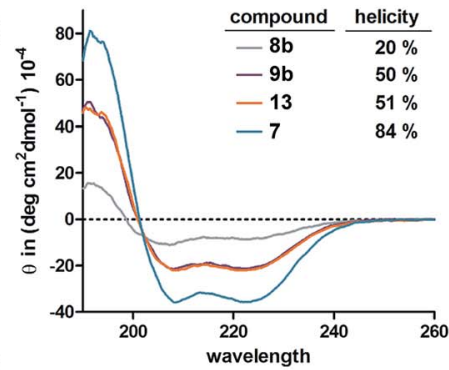

e)

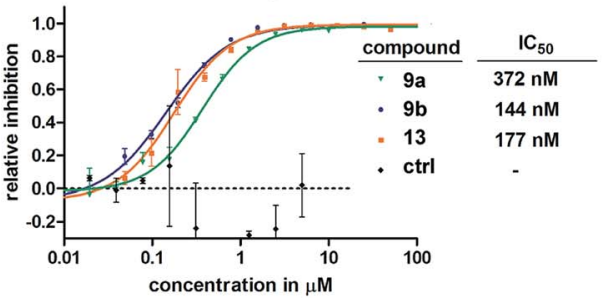

f)

13-CF

7-CF b)
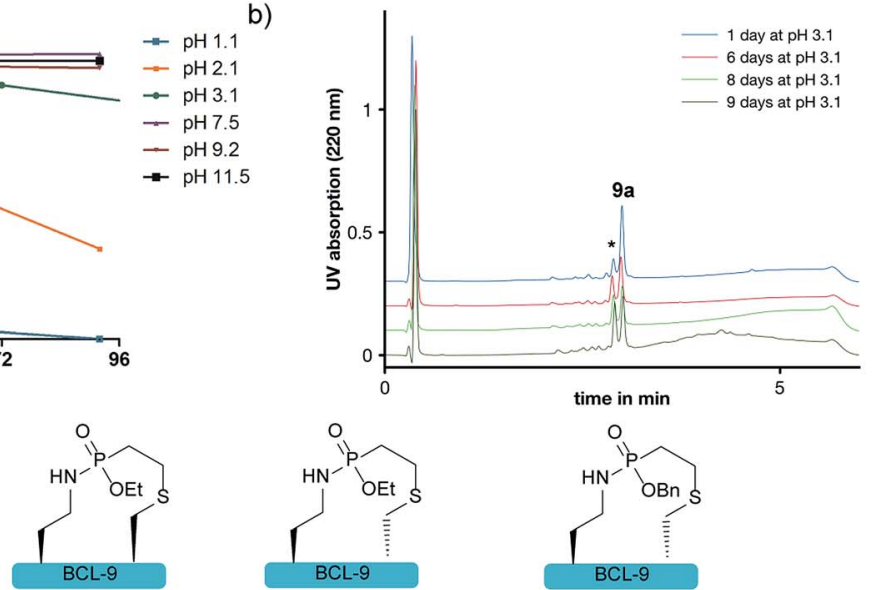

9a, $45 \%$

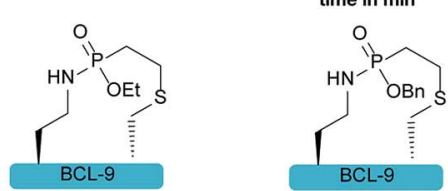

9 b, $43 \%$
13, $33 \%$

$2 \mathrm{sec}$

$10 \mathrm{sec}$

$20 \mathrm{sec}$

9b-CF
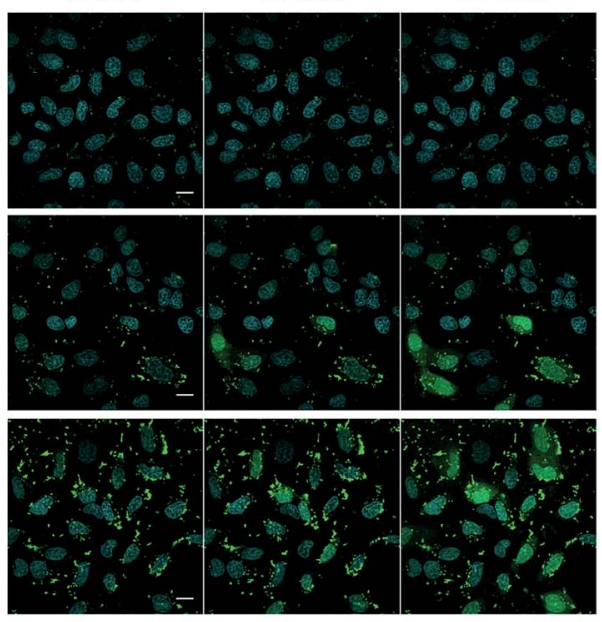

Fig. 4 (a) Stability of 9a at different $\mathrm{pH}$ values. Shown are mean and error from three independent measurements, monitored by UPLC-UV. (b) UPLC-UV spectra of 9 a upon incubation at pH 3.1 over several days. *P-N-bond cleavage is the only degradation product observed. (c) Structures of differently cyclized BCL-9 peptides. Stated are isolated yields from the cyclization reaction. (d) CD-measurement of peptides. (e) HTRF assay shows disruption of PPI by peptidic inhibitors. (f) Cellular uptake studies of $10 \mu \mathrm{M}$ mixture of $10 \%$ carboxy-fluorescein (CF)-labelled and $90 \%$ non-labelled peptides in HeLa cells. Confocal images with scale bar $=20 \mu \mathrm{m}$. Depicted is the overlay of fluorescein-fluorescence and Hoechst nucleus stain at different time points of laser $(405 / 488 \mathrm{~nm})$ irradiation.

illustrates the modularity of our method that allows a facile exchange of the $O$-substituent of phosphonamidates to modulate the functional properties of the peptide ligation products.

In addition to the side-chain-stapled peptides, we further expanded our novel cyclization method to a more general macrocyclization technique for enhancing the cellular uptake properties of protein conjugates. Therefore, we aimed for the cyclization of an $R_{10}$ peptide, which is, like other arginine-rich peptides, able to enhance the transduction of functional proteins into living cells when covalently attached to the protein cargo. ${ }^{48}$ We and others observed that the cellular transduction behavior is significantly improved for the cyclic version of $\mathrm{cR}_{10}$ when compared to its linear counterpart. ${ }^{22,49,50}$ As an alternative to the previously applied cyclized $\mathrm{R}_{10}$-CPP 14 , we synthesized peptide 15 containing a cysteine and an azidohomoalanine residue to replace the former lactam bridge after our novel cyclization technique. The linear unprotected peptide was reacted with the crude diethylvinylphosphonite (route III) in dry DMSO overnight to yield $48 \%$ of the in situ macrocyclized peptide $\mathbf{1 6}$ after purification. For conjugation of $\mathbf{1 6}$ to a protein cargo, the $N$-terminus of the peptide was capped with an ethynylphosphonamidate-NHS building block $17^{31}$ to yield 18 (Fig. 5a) for Cys-selective labeling reactions. Conjugation to eGFP that carries a single addressable cysteine proceeded quantitatively according to MALDI-TOF-MS (Fig. 5b). Cellular uptake of the protein was demonstrated by confocal fluorescence imaging after incubation of HeLa-cells with $30 \mu \mathrm{M}$ conjugate for one hour at $37{ }^{\circ} \mathrm{C}$ in HEPES buffer and was compared to that of the standard lactam cyclized $\mathrm{cR}_{10}$-eGFP conjugate. Cytosolic and nucleolar GFP fluorescence was observed and both proteinpeptide-conjugates showed the same amount of transduced cells, indicating the feasibility of using our novel cyclization method as a macrocyclization technique (Fig. 5c). 
a)

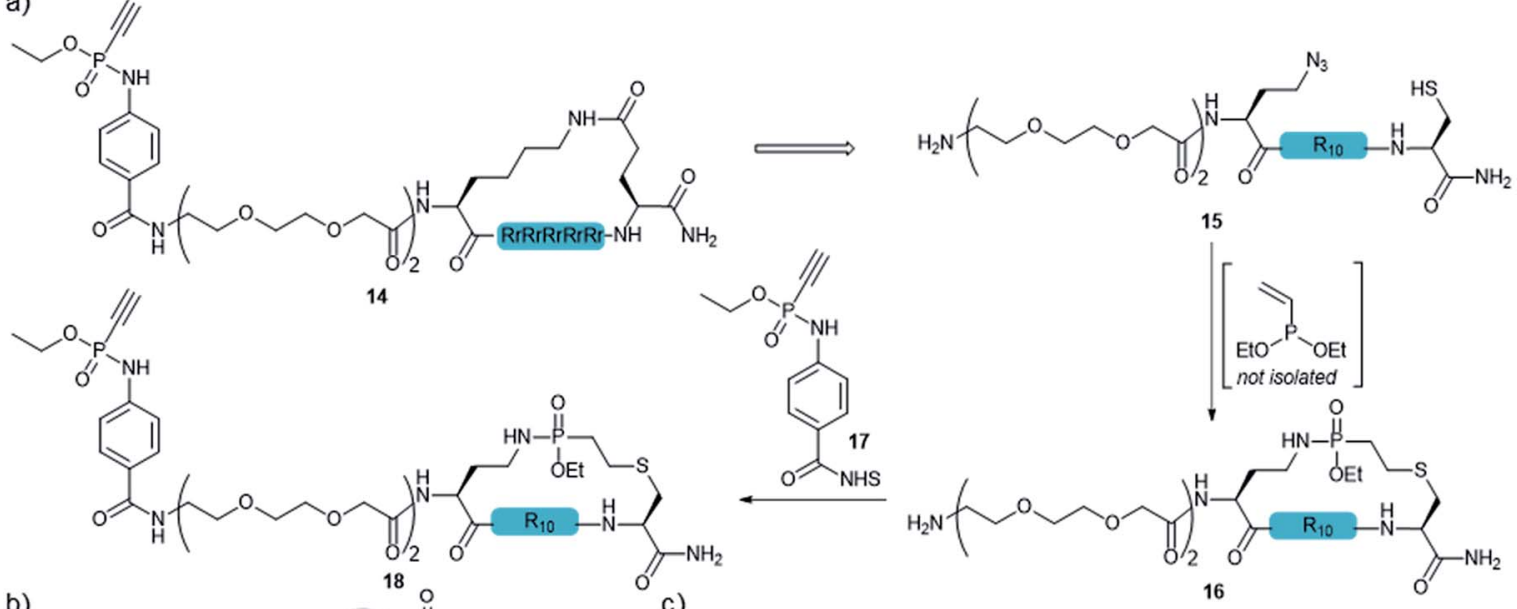

b)

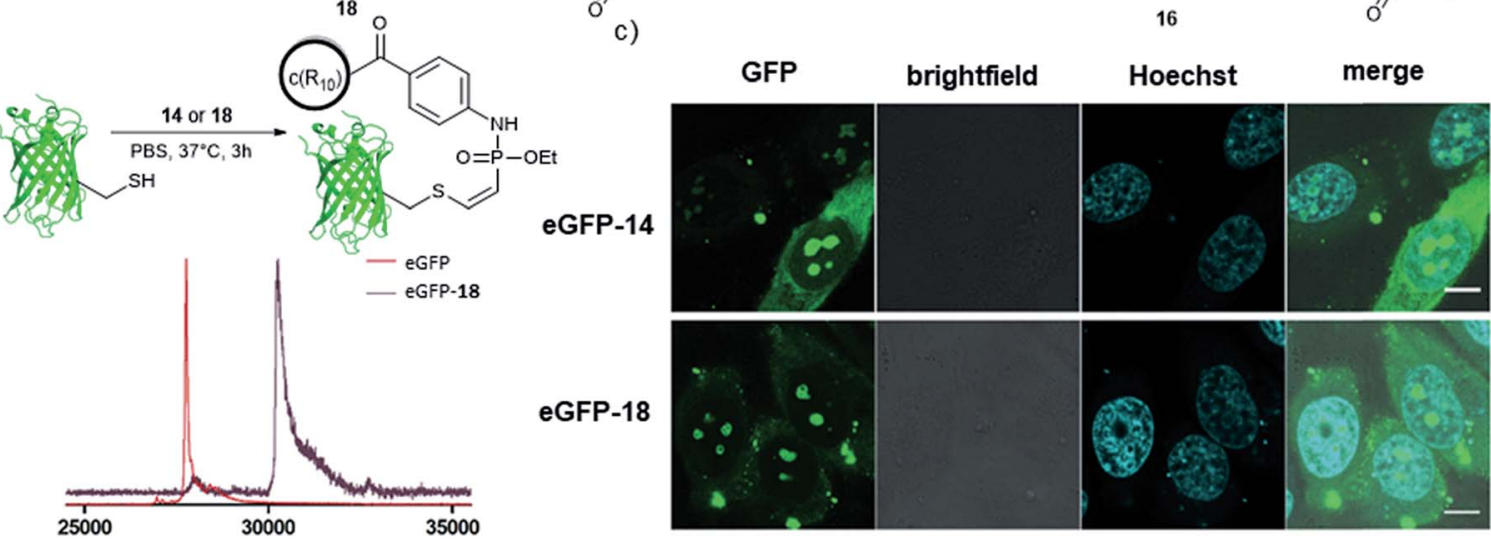

Fig. 5 (a) Principle of the Staudinger induced peptide cyclization with $\mathrm{R}_{10}$ and subsequent attachment of a thiol reactive handle. (b) Conjugation of $\mathrm{CR}_{10}$ to eGFP C70M S147C. Shown are MALDI-TOF-MS spectra before and after the reaction with 23 . (c) Cellular uptake studies of $30 \mu \mathrm{M}$ eGFP-14 and eGFP-18 in HeLa cells. Confocal images with scale bar $=10 \mu \mathrm{m}$.

\section{Conclusions}

In summary, we introduce the SPhR with vinylphosphonites as a versatile tool for the chemoselective cyclization of peptides. Furthermore, we demonstrate that variation of the phosphonite's $O$-substituent enables fine-tuning of the electrophilicity for intermolecular protein and antibody labeling. Additionally, this variation facilitates the incorporation of functional handles that can modulate the properties of the reaction product as demonstrated for the increased cellular uptake of phosphonamidatecyclized BCL9 peptides. Finally, the high yielding peptide cyclization technique not only allows facile stapling of peptides but furthermore also facilitates a general macrocyclization strategy as shown for cCPPs. We believe that this tool will enable researchers to develop both novel cysteine reactive probes for applications where a narrow reactivity window is crucial and cyclic peptides with enhanced pharmacological properties that can be manipulated by phosphonamidate ester variation.

\section{Conflicts of interest}

The authors declare competing financial interests. The technology described in the manuscript is part of a pending patent application by M.-A. K., M. G. and C. P. R. H.

\section{Acknowledgements}

We thank H. Stephanowitz and Dr Fan Liu for performing tandem MS experiments, K. K. Hassanin for excellent technical assistance and Dr K. Licha for the provision of Cetuximab. This work was supported by grants from the Deutsche Forschungsgemeinschaft (SPP1623) to C. P. R. H. (HA 4468/9-1), the Einstein Foundation Berlin (Leibniz-Humboldt Professorship), the Boehringer-Ingelheim Foundation (Plus 3 award), the Leibniz Association with the Leibniz competition to C. P. R. H. and the Fonds der Chemischen Industrie to C. P. R. H.

\section{Notes and references}

1 S. B. Gunnoo and A. Madder, ChemBioChem, 2016, 17, 529-553. 2 J. M. Chalker, G. J. L. Bernardes, Y. A. Lin and B. G. Davis, Chem.-Asian J., 2009, 4, 630-640.

3 S. O. Doronina, B. E. Toki, M. Y. Torgov, B. A. Mendelsohn, C. G. Cerveny, D. F. Chace, R. L. DeBlanc, R. P. Gearing, T. D. Bovee, C. B. Siegall, J. A. Francisco, A. F. Wahl, D. L. Meyer and P. D. Senter, Nat. Biotechnol., 2003, 21, 778-784.

$4 \mathrm{~J}$. Bertran-Vicente, M. Penkert, O. Nieto-Garcia, J.-M. Jeckelmann, P. Schmieder, E. Krause and C. P. R. Hackenberger, Nat. Commun., 2016, 7, 12703. 
5 B. F. Cravatt, A. T. Wright and J. W. Kozarich, Annu. Rev. Biochem., 2008, 77, 383-414.

6 J. E. Moore and W. H. Ward, J. Am. Chem. Soc., 1956, 78, 2414-2418.

7 S. Brocchini, S. Balan, A. Godwin, J.-W. Choi, M. Zloh and S. Shaunak, Nat. Protoc., 2006, 1, 2241-2252.

8 B. Bernardim, P. M. S. D. Cal, M. J. Matos, B. L. Oliveira, N. Martínez-Sáez, I. S. Albuquerque, E. Perkins, F. Corzana, A. C. B. Burtoloso, G. Jiménez-Osés and G. J. L. Bernardes, Nat. Commun., 2016, 7, 13128.

9 D. R. Goddard and L. Michaelis, J. Biol. Chem., 1935, 112, 361-371.

10 D. Abegg, R. Frei, L. Cerato, D. Prasad Hari, C. Wang, J. Waser and A. Adibekian, Angew. Chem., 2015, 127, 11002-11007.

11 J. Václavík, R. Zschoche, I. Klimánková, V. Matoušek, P. Beier, D. Hilvert and A. Togni, Chem.-Eur. J., 2017, 23, 6490-6494.

12 C. Zhang, M. Welborn, T. Zhu, N. J. Yang, M. S. Santos, T. Van Voorhis and B. L. Pentelute, Nat. Chem., 2015, 8, 120.

13 E. V. Vinogradova, C. Zhang, A. M. Spokoyny, B. L. Pentelute and S. L. Buchwald, Nature, 2015, 526, 687.

14 M. J. Niphakis and B. F. Cravatt, Annu. Rev. Biochem., 2014, 83, 341-377.

15 R. Lonsdale and R. A. Ward, Chem. Soc. Rev., 2018, 47, 38163830 .

16 K. T. Barglow and B. F. Cravatt, Chem. Biol., 2004, 11, 15231531.

17 M. E. Otero-Ramirez, T. Passioura and H. Suga, Biomedicines, 2018, 6, 116.

18 A. Celine and S. Claudio, Curr. Med. Chem., 2002, 9, 963-978.

19 L. D. Walensky, A. L. Kung, I. Escher, T. J. Malia, S. Barbuto, R. D. Wright, G. Wagner, G. L. Verdine and S. J. Korsmeyer, Science, 2004, 305, 1466-1470.

20 L. D. Walensky and G. H. Bird, J. Med. Chem., 2014, 57, 62756288.

21 L. J. Walport, R. Obexer and H. Suga, Curr. Opin. Biotechnol., 2017, 48, 242-250.

22 G. Lättig-Tünnemann, M. Prinz, D. Hoffmann, J. Behlke, C. Palm-Apergi, I. Morano, H. D. Herce and M. C. Cardoso, Nat. Commun., 2011, 2, 453.

23 S. Kalhor-Monfared, M. R. Jafari, J. T. Patterson, P. I. Kitov, J. J. Dwyer, J. M. Nuss and R. Derda, Chem. Sci., 2016, 7, 3785-3790.

24 A. M. Spokoyny, Y. Zou, J. J. Ling, H. Yu, Y.-S. Lin and B. L. Pentelute, J. Am. Chem. Soc., 2013, 135, 5946-5949.

25 C. Heinis, T. Rutherford, S. Freund and G. Winter, Nat. Chem. Biol., 2009, 5, 502.

26 S. S. Kale, C. Villequey, X.-D. Kong, A. Zorzi, K. Deyle and C. Heinis, Nat. Chem., 2018, 10, 715-723.

27 N. A. Afagh and A. K. Yudin, Angew. Chem., Int. Ed., 2010, 49, 262-310.

28 L. Peng and P. R. Peter, Curr. Top. Med. Chem., 2002, 2, 325341.

29 M. Pelay-Gimeno, T. Bange, S. Hennig and T. N. Grossmann, Angew. Chem., Int. Ed., 2018, 57, 11164-11170.
30 M. R. J. Vallée, P. Majkut, I. Wilkening, C. Weise, G. Müller and C. P. R. Hackenberger, Org. Lett., 2011, 13, 5440-5443.

31 M.-A. Kasper, M. Glanz, A. Stengl, M. Penkert, S. Klenk, T. Sauer, D. Schumacher, J. Helma, E. Krause, M. C. Cardoso, H. Leonhardt and C. Hackenberger, Angew. Chem., Int. Ed., DOI: 10.1002/anie.201814715.

32 R. Kleineweischede and C. P. R. Hackenberger, Angew. Chem., Int. Ed., 2008, 47, 5984-5988.

33 T. Fukuzumi, L. Ju and J. W. Bode, Org. Biomol. Chem., 2012, 10, 5837-5844.

34 H. Y. Lam, Y. Zhang, H. Liu, J. Xu, C. T. T. Wong, C. Xu and X. Li, J. Am. Chem. Soc., 2013, 135, 6272-6279.

35 F. Gao, X. Yan and K. Auclair, Chem.-Eur. J., 2009, 15, 20642070.

36 K. D. Siebertz and C. P. R. Hackenberger, Chem. Commun., 2018, 54, 763-766.

37 M. R. J. Vallée, L. M. Artner, J. Dernedde and C. P. R. Hackenberger, Angew. Chem., Int. Ed., 2013, 52, 9504-9508.

38 F. Saito, H. Noda and J. W. Bode, ACS Chem. Biol., 2015, 10, 1026-1033.

39 Q. Luo, Y. Tao, W. Sheng, J. Lu and H. Wang, Nat. Commun., 2019, 10, 142.

40 J. F. Ponte, X. Sun, N. C. Yoder, N. Fishkin, R. Laleau, J. Coccia, L. Lanieri, M. Bogalhas, L. Wang, S. Wilhelm, W. Widdison, J. Pinkas, T. A. Keating, R. Chari, H. K. Erickson and J. M. Lambert, Bioconjugate Chem., 2016, 27, 1588-1598.

41 M. L. Nielsen, M. Vermeulen, T. Bonaldi, J. Cox, L. Moroder and M. Mann, Nat. Methods, 2008, 5, 459-460.

42 C. F. Brewer and J. P. Riehm, Anal. Biochem., 1967, 18, 248255.

43 K. Takada, D. Zhu, G. H. Bird, K. Sukhdeo, J.-J. Zhao, M. Mani, M. Lemieux, D. E. Carrasco, J. Ryan, D. Horst, M. Fulciniti, N. C. Munshi, W. Xu, A. L. Kung, R. A. Shivdasani, L. D. Walensky and D. R. Carrasco, Sci. Transl. Med., 2012, 4, 148 ra117.

44 I. Wilkening, G. del Signore and C. P. R. Hackenberger, Chem. Commun., 2008, 2932-2934.

45 A. Klaus and W. Birchmeier, Nat. Rev. Cancer, 2008, 8, 387.

46 G. L. Verdine and G. J. Hilinski, in Methods Enzymol, ed. K. D. Wittrup and G. L. Verdine, Academic Press, 2012, vol. 503, pp. 3-33.

47 K. Sakagami, T. Masuda, K. Kawano and S. Futaki, Mol. Pharm., 2018, 15, 1332-1340.

48 N. Nischan, H. D. Herce, F. Natale, N. Bohlke, N. Budisa, M. C. Cardoso and C. P. R. Hackenberger, Angew. Chem., Int. Ed., 2015, 54, 1950-1953.

49 H. D. Herce, D. Schumacher, A. F. L. Schneider, A. K. Ludwig, F. A. Mann, M. Fillies, M.-A. Kasper, S. Reinke, E. Krause, H. Leonhardt, M. C. Cardoso and C. P. R. Hackenberger, Nat. Chem., 2017, 9, 762-771.

50 A. F. L. Schneider, A. L. D. Wallabregue, L. Franz and C. P. R. Hackenberger, Bioconjugate Chem., 2019, 30, 400404. 